\title{
Human papillomavirus: footprints in the population of western India
}

\author{
Ashi Robert Thobias ${ }^{1,2}$, Kinjal Ankit Patel', Supreet Dhananjay Bhatt ${ }^{3}$, \\ Kruti Ashvinkumar Mehta ${ }^{1,2}$, Chetana Deepal Parekh ${ }^{4}$, Pariseema Sharad Dave ${ }^{4}$, \\ Prabhudas Shankarbhai Patel ${ }^{1,2}$ \\ 'Biology Department, Gujarat Cancer and Research Institute, Ahmedabad, India; ${ }^{2}$ Gujarat University Ahmedabad, India; ${ }^{3}$ Surgical Oncology \\ Department, Gujarat Cancer and Research Institute, Ahmedabad, India; ${ }^{4}$ Department of Gynecological Oncology, Gujarat Cancer and Research \\ Institute, Ahmedabad, India
}

OBJECTIVES: Cancer is a multi-factorial disease, with various intrinsic and environmental factors contributing to its occurrence. Human papillomavirus (HPV) has been associated with the occurrence of many cancers. India severely suffers from 3 HPV-associated cancers (cervical cancer, oral cancer, and oropharyngeal cancer). Hence, the present study aimed to evaluate the HPV burden in these 3 cancers among patients from the western region of India.

METHODS: DNA was isolated from samples from 400 cervical cancer, 127 oral cancer, and 75 oropharyngeal cancer patients. Polymerase chain reaction was performed using degenerate primers for HPV infection.

RESULTS: Overall, HPV infection was observed in $87 \%$ of cervical cancer cases, $12.5 \%$ of oral cancer cases, and $26.7 \%$ of oropharyngeal cancer cases when analyzed with a cumulative detection method using the MY 09/11, GP 5+/6+, and CP I/II primer sets.

CONCLUSIONS: A significant prevalence of HPV infection was detected in all 3 cancers using the degenerate primer sets. This finding implies that testing for HPV infection using multiple primer sets is crucial for determining its actual prevalence in various malignancies.

KEY WORDS: Oropharyngeal neoplasms, Oral neoplasms, Cervical neoplasms, Human papilloma virus

\section{INTRODUCTION}

Human papillomavirus (HPV) has been identified as an etiological factor for cancer that contributes to 600,000 cancer cases every year worldwide [1]. HPV has been associated with cancer since the 1970s, when the scientist Zur Hausen discovered the

Correspondence: Prabhudas Shankarbhai Patel

Biology Department, Gujarat Cancer and Research Institute, Ahmedabad 380 016, India

E-mail: prabhudas_p@hotmail.com

Received: Nov 6, 2020 / Accepted: Feb 3, 2021 / Published: Feb 3, 2021

This article is available from: https://e-epih.org/

(C) This is an open-access article distributed under the terms of the Creative Commons Attribution License (https://creativecommons.org/licenses/by/4.0/), which permits unrestricted use, distribution, and reproduction in any medium, provided the original work is properly cited.

(C) 2021, Korean Society of Epidemiology presence of HPV in genital warts and cervical cancer [2]. Subsequently, persistent HPV infection has been documented in patients with cervical, vaginal, penile, vulvar, anal, rectal, and oropharyngeal cancer $[3,4]$. HPV-associated cancers are known to show different clinical, pathological, molecular, and epidemiological profiles as compared to their counterparts. They also have a varied response to therapy and show an overall better survival rate [5-7]. Thus, it is imperative to study the prevalence of these cancers. A standard protocol for the detection of HPV has not yet been defined, which has generated doubts about when and why to assess HPV status [8]. Most studies on the prevalence of HPV have focused on the highly prevalent high-risk human papillomavirus (HR-HPV) types 16 and 18. However, other HPV types also show oncogenic potential and should be evaluated. Furthermore, regional variation has also been observed in HPV prevalence [9].

Degenerate primer sets, including MY 09/11 and GP 5+/6+, are specifically designed in tandem with the highly conserved L1 
Table 1. Primer sequence, amplicon size and region specificity of degenerative primer sets

\begin{tabular}{lccc}
\hline Variables & MY 09/11 & GP 5+/6+ & CP I/II \\
\hline Sequence & & & \\
$\quad$ Forward & 5'-CGT CCM ARR GGA WAC TGA TC-3' & 5'-TTT GTT ACT GTG GTA GAT ACT AC-3' & 5'-TTA TCW TAT GCC CAY TGT ACC AT-3' \\
Reverse & $5^{\prime}$-GCM CAG GGW CAT AAY AAT GG-3' & 5'-GAA AAA TAA ACT GTA AAT CAT ATT C-3' & 5'-ATG TTA ATW SAG CCW CCA AAA TT-3' \\
Amplicon size (bp) & 450 & 150 & 180 \\
HPV region & L1 & L1 & E1 \\
\hline
\end{tabular}

HPV, human papillomavirus.

region of the HPV genome, whereas CP I/II is designed for the E1 region. The degenerate primers were shown to have an improved HPV detection rate in our previous study [10]. Even though the prevalence of HPV-associated cancers, such as cervical cancer, is high in India, countrywide data on HPV infection and genotype distribution are not available, although this information would be useful for a wider vaccination program [11]. Furthermore, there has been an increase of HPV-positive cases of head and neck cancers in the past few decades [12-14]. These cancers are among the major malignancies ailing India [15]. Moreover, there is a dearth of evidence regarding HPV prevalence in western India.

Hence the present study aimed to evaluate the prevalence of HPV in cervical cancer, oral cancer, and oropharyngeal cancer patients in the western region of India. Degenerate primer sets were used for HPV detection, with the specific aim of detecting HPV infection of any strain.

\section{MATERIALS AND METHODS}

The present study evaluated the burden of HPV infection in the 3 most prevalent HPV-associated cancers (i.e., cervical cancer, oral cancer, and oropharyngeal cancer) using degenerate primer sets.

\section{Sample collection}

The patients were enrolled in the study after histopathological confirmation from the pathology department at the Gujarat Cancer and Research Institute, Ahmedabad. Tissue biopsy samples of 400 cervical cancer, 127 oral cancer, and 75 oropharyngeal cancer patients were collected for the study after informed consent. The patients did not undergo any prior treatment for the malignancy. Patients with a simultaneous diagnosis of other major illness or coinfections with human immunodeficiency virus, hepatitis $\mathrm{C}$ virus, and hepatitis B virus were excluded from the study. Immediately after collection, the tissue samples were washed with phosphate-buffered saline ( $\mathrm{pH} 7.4$ ) and promptly stored at $-80^{\circ} \mathrm{C}$ until analysis.

\section{Detection of human papillomavirus infection}

DNA was isolated from tissues using a commercially available DNA isolation kit (Qiagen, Valencia, CA, USA). The DNA yield was checked by spectrophotometric analysis (Shimadzu UV-1800, Shimadzu Inc., Kyoto, Japan) and DNA integrity was checked by
Table 2. Clinical and clinicopathological details of the cervical cancer cohort

\begin{tabular}{lc}
\hline Characteristics & No. of patients (\%) \\
\hline Age (yr) & \\
$<50$ & $233(58.3)$ \\
$\geq 50$ & $167(41.8)$ \\
Menopause status & \\
Premenopausal & $90(22.5)$ \\
Postmenopausal & $185(46.3)$ \\
Unknown & $125(31.2)$ \\
Histology & \\
Squamous cell carcinoma & $365(91.2)$ \\
Adenocarcinoma & $30(7.5)$ \\
Unknown & $5(1.2)$ \\
Type of growth & \\
Exophytic & $103(25.7)$ \\
Infiltrative & $94(23.5)$ \\
Unknown & $203(50.7)$ \\
Differentiation & \\
Well & $14(3.5)$ \\
Moderate & $254(63.5)$ \\
Poor & $75(18.7)$ \\
Unknown & $57(14.2)$ \\
Stage & \\
I & $31(7.8)$ \\
II & $62(15.5)$ \\
III & $234(58.5)$ \\
Unknown & $11(2.7)$ \\
Recurrence & $62(15.5)$ \\
Yes & $16(4.0)$ \\
No & $212(53.0)$ \\
Unknown & $172(43.0)$ \\
\hline &
\end{tabular}

agarose gel electrophoresis using an $0.8 \%$ gel.

The DNA was amplified using the degenerate primer sets MY 09/11, GP 5+/6+, and CP I/II separately on a thermal cycler (Proflex PCR System, Life Technologies, Carlsbad, CA, USA). The polymerase chain reaction (PCR) was prepared using a previously standardized protocol [10].

For the MY 09/11 primers, PCR was carried out as follows: initial denaturation at $95^{\circ} \mathrm{C}$ for 3 minutes, followed by 40 cycles of 
Table 3. Clinical and clinicopathological details of the oral cancer cohort

\begin{tabular}{lc}
\hline Characteristics & No. of patients (\%) \\
\hline Age (yr) & \\
$<45$ & $59(46.5)$ \\
$\geq 45$ & $68(53.5)$ \\
Sex & \\
Male & $105(82.7)$ \\
Female & $22(17.3)$ \\
Site of origin & \\
Buccal & $48(37.8)$ \\
Tongue & $43(33.8)$ \\
Other & $18(14.2)$ \\
Mixed & $18(14.2)$ \\
Tobacco habit & \\
Yes & $106(83.46)$ \\
No & $21(16.54)$ \\
Histology & \\
Squamous cell carcinoma & $124(97.6)$ \\
Verrucous carcinoma & $2(1.6)$ \\
Unknown & $1(0.8)$ \\
Differentiation & \\
Well & $37(29.1)$ \\
Moderate & $78(61.4)$ \\
Poor & $5(3.9)$ \\
Unknown & $7(5.6)$ \\
Stage & \\
I & $13(10.2)$ \\
III & $45(35.4)$ \\
IV & $13(10.2)$ \\
Unknown & $51(40.2)$ \\
Nymph node metastasis & $5(4.0)$ \\
\hline & \\
\hline & \\
Unknown & $(3.2)$ \\
\hline
\end{tabular}

denaturation at $95^{\circ} \mathrm{C}$ for 1 minute, annealing at $49.3^{\circ} \mathrm{C}$ for $1 \mathrm{~min}$ ute, and extension at $72^{\circ} \mathrm{C}$ for 1 minute. In the final cycle, extension was carried out for 10 minutes. For the GP $5+/ 6+$ primers, PCR was carried out as follows: initial denaturation at $95^{\circ} \mathrm{C}$ for $3 \mathrm{~min}-$ utes, followed by 40 cycles of denaturation at $95^{\circ} \mathrm{C}$ for 1 minute, annealing at $48^{\circ} \mathrm{C}$ for 1 minute, and extension at $72^{\circ} \mathrm{C}$ for $1 \mathrm{~min}$ ute. In the final cycle, extension was carried out for 10 minutes. For the CP I/II primers, PCR was carried out as follows: initial denaturation at $95^{\circ} \mathrm{C}$ for 3 minutes, followed by 40 cycles of denaturation at $95^{\circ} \mathrm{C}$ for 1 minute, annealing at $56.5^{\circ} \mathrm{C}$ for 1 minute, and extension at $72^{\circ} \mathrm{C}$ for 1 minute. In the final cycle, extension was carried out for 10 minutes. The samples were checked for the presence of HPV infection using 2\% agarose gel electrophoresis.

The MY 09/11 and GP 5+/6+ primers are designed to detect the $\mathrm{L} 1$ region of a wide range of the HPV genome, while CP I/II
Table 4. Clinical and clinicopathological details of the oropharyngeal cancer cohort

\begin{tabular}{lc}
\hline Characteristics & No. of patients (\%) \\
\hline Age (yr) & \\
$<57$ & $38(50.6)$ \\
$\geq 57$ & $37(49.3)$ \\
Sex & \\
Male & $74(98.6)$ \\
Female & $1(1.4)$ \\
Site of initiation & \\
Base of tongue & $30(40.0)$ \\
Valeculla & $4(5.3)$ \\
Tonsillar fossa & $10(13.3)$ \\
Mixed site & $5(6.7)$ \\
Other site & $26(34.7)$ \\
Tobacco habit & \\
Yes & $66(88.0)$ \\
No & $1(1.3)$ \\
Unknown & $8(10.7)$ \\
Histology & \\
Squamous cell carcinoma & $66(88.0)$ \\
Unknown & $9(12.0)$ \\
Tumor differentiation & \\
Well & $6(8.0)$ \\
Moderate & $42(56.0)$ \\
Poor & $11(14.6)$ \\
Unknown & $16(21.4)$ \\
\hline & \\
\hline
\end{tabular}

detects the E1 region (Table 1). A combination of these primer sets may curb the rate of false negativity for HPV infection.

\section{Ethics statement}

The study was approved by the Gujarat Cancer and Research Institute/the Gujarat Cancer Society Ethics Committee (approval no. EC/31/2018). Informed consent was confirmed by the committee.

\section{RESULTS}

\section{Clinicopathological features of the study cohort}

The present study was carried out in 3 highly prevalent HPVassociated cancers (i.e., cervical cancer, oral cancer, and oropharyngeal cancer). The clinical and clinicopathological parameters of the cervical cancer patients $(n=400)$, oral cancer patients $(\mathrm{n}=127)$, and oropharyngeal cancer patients $(\mathrm{n}=75)$ are given in Tables 2-4, respectively.

\section{Prevalence of human papillomavirus infection by degenerate primer sets}

All 3 cohorts were screened for comprehensive HPV infections using the degenerate primer sets MY 09/11, GP 5+/6+ and CP I/II. Representative PCR patterns are depicted in Figure 1. 

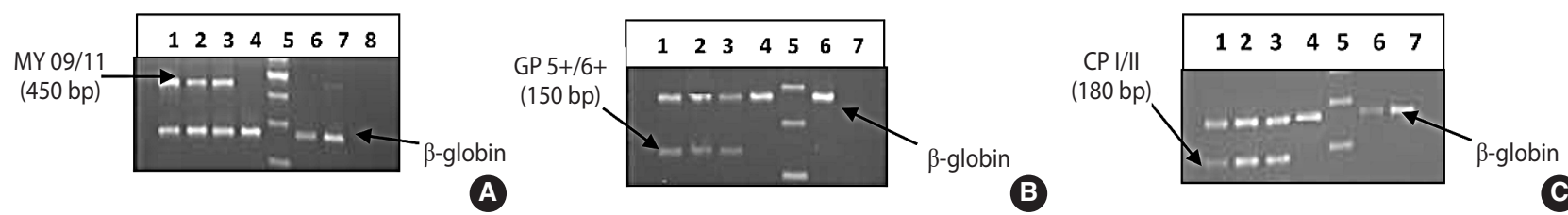

Figure 1. Representative patterns of gel electrophoresis of polymerase chain reaction by degenerative primers (A) MY 09/11, (B) GP 5+/6+, and $(\mathrm{C}) \mathrm{CPI} / \mathrm{II}$.
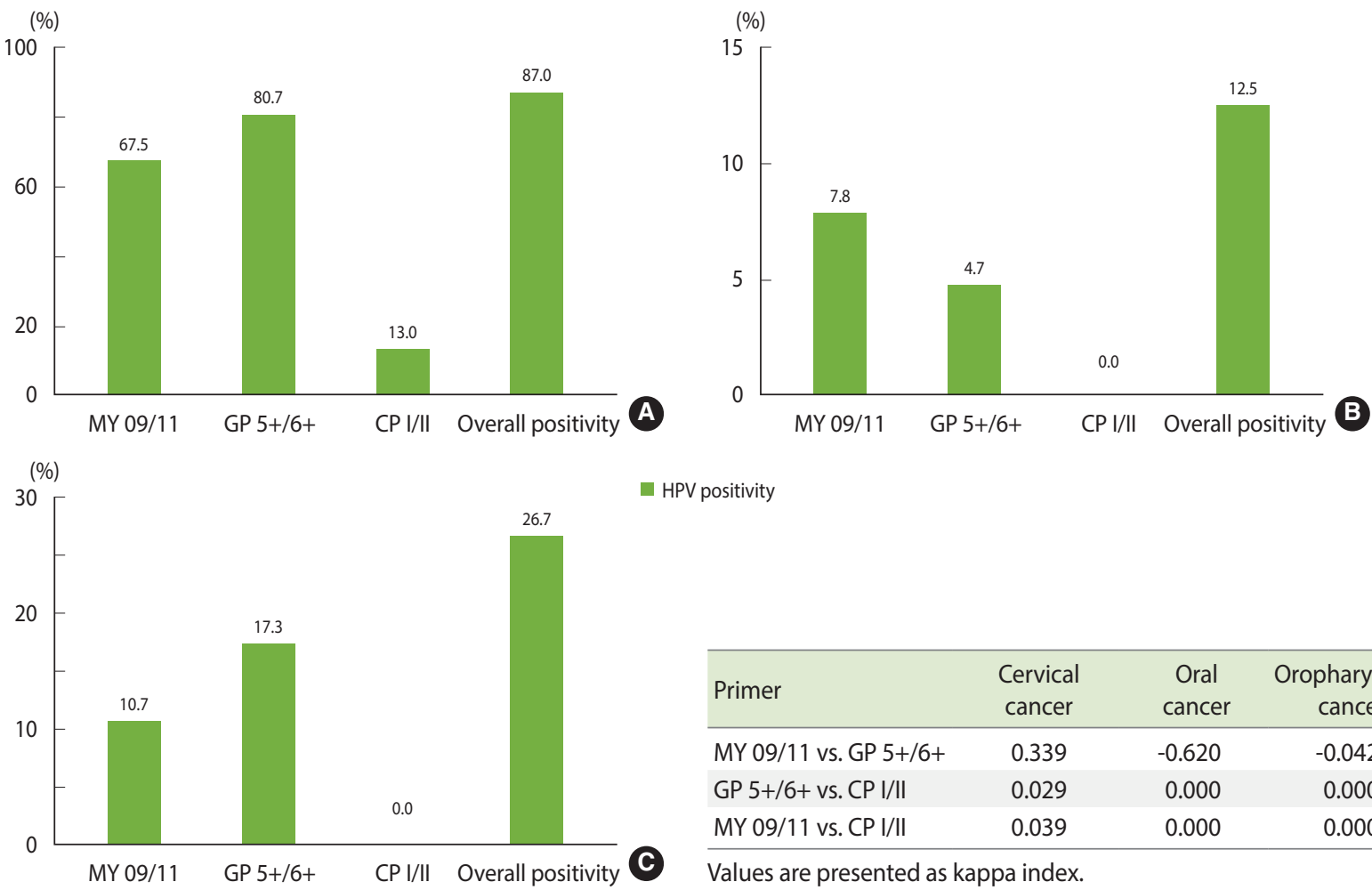

\begin{tabular}{lccc}
\hline Primer & $\begin{array}{c}\text { Cervical } \\
\text { cancer }\end{array}$ & $\begin{array}{c}\text { Oral } \\
\text { cancer }\end{array}$ & $\begin{array}{c}\text { Oropharyngeal } \\
\text { cancer }\end{array}$ \\
\hline MY 09/11 vs. GP 5+/6+ & 0.339 & -0.620 & -0.042 \\
GP 5+/6+ vs. CP I/II & 0.029 & 0.000 & 0.000 \\
MY 09/11 vs. CP I/II & 0.039 & 0.000 & 0.000 \\
\hline Values are presented as kappa index. & &
\end{tabular}

Figure 2. Human papillomavirus (HPV) positivity by degenerative primer sets in (A) cervical cancer, (B) oral cancer, and (C) oropharyngeal cancer.

\section{Cervical cancer}

Out of the cervical cancer patients enrolled in the study, $67.5 \%$ were positive for HPV infection by the MY 09/11 primer set, $80.7 \%$ of cases were positive by the GP $5+/ 6+$ primer set, and $13.0 \%$ of cases were positive by the CP I/II primer set (Figure $2 \mathrm{~A}$ ). The cumulative HPV infection rate detected in the cohort by the combination of all 3 degenerate primer sets was $87.0 \%$ (Figure 2A). There was marginal agreement between the detection of HPV by the MY 09/11 and GP 5+/6+ primer sets (kappa index=0.339), whereas the MY 09/11 and CP I/II primer sets (kappa index= 0.039 ) and the GP 5+/6+ and CP I/II primer sets (kappa index = 0.029 ) were not in agreement (Figure 2).

\section{Oral cancer}

In the investigation of 127 oral cancer cases, we observed that $7.8 \%$ of cases were positive for HPV infection by MY 09/11, while $4.7 \%$ of cases were positive by GP $5+/ 6+$ and all cases were nega- tive for HPV infection by the CP I/II primer set (Figure 2B). The total HPV positivity rate according to the combined investigation using the degenerate primer sets was $12.5 \%$ (Figure $2 \mathrm{~B}$ ). There was no agreement in the detection of HPV between the MY 09/11 and GP $5+/ 6+$ primer sets (kappa index $=-0.620$ ), between the MY 09/11 and CP I/II primer sets (kappa index $=0.000$ ), and between the GP $5+/ 6+$ and CP I/II primer sets (kappa index $=0.000$ ) (Figure 2).

\section{Oropharyngeal cancer}

Among 75 oropharyngeal cancer cases, HPV positivity was found in $10.7 \%$ of cases using the MY $09 / 11$ primer set and in $17.3 \%$ of cases using the GP 5+/6+ primers. Similar to oral cancer, no positivity was observed using the CP I/II primer set (Figure 2C). A total HPV positivity rate of $26.7 \%$ was observed in the cohort (Figure $2 \mathrm{C}$ ). There was no agreement in the detection of HPV between the MY 09/11 and GP 5+/6+ primer sets (kappa index=-0.042), 
between the MY 09/11 and CP I/II primer sets (kappa index= 0.000), and between the GP $5+/ 6+$ and CP I/II primer sets (kappa index $=0.000)$ (Figure 2).

\section{DISCUSSION}

The present study evaluated the burden of HPV infection in the 3 most prevalent HPV-associated cancers (i.e., cervical cancer, oral cancer, and oropharyngeal cancer). The study was carried out in patients from the western region of India using degenerate primer sets. Our study demonstrated a comprehensive screening module to detect HPV infections without exclusion of any HPV type.

We observed that $67.5 \%$ of cervical cancer cases were positive for HPV infection using the MY 09/11 primer set. Similar results were obtained in a study conducted in Odisha, which showed a $60.3 \%$ infection rate when analyzed using the PGMY 09/11 primer sets [11]. In contrast, a multi-center study from India showed HPV infection in $83.4 \%$ of cervical cancer specimens [16]. In this cohort, $80.7 \%$ of cases were positive using the GP $5+/ 6+$ primer set. A study from central India reported that $93.3 \%$ of cases were positive using the GP 5+/6+ primer set [17], while a study conducted in Karachi, Pakistan showed only a $27.3 \%$ prevalence rate in females with cervical abnormalities [18]. When analyzed with the CP I/II primer set, $13 \%$ of cases were positive for infection, while the total positivity observed with respect to all 3 degenerate primer sets was $87.0 \%$, which is higher than was reported in Tamil Nadu using the MY 09/11 and GP 5+/6+ primer sets in a study that found an overall HPV infection rate of 54.9\% [19]. Moreover, studies from India have reported varying prevalence rates (15-85\%) of HPV infection in cervical cancer patients [20].

In the investigation of 127 oral cancer cases, we observed that $7.8 \%$ of cases were positive for HPV infection by the MY 09/11 primer set, while $4.7 \%$ of cases were positive by the GP $5+/ 6+$ primer set. This rate is lower than that reported in another study that recorded an HPV prevalence of $35.8 \%$ in oral mucosa samples when analyzed with the GP $5+/ 6+$ primer set, whereas a positivity rate of only $2.2 \%$ was reported using the MY 09/11 primer set [21]. Furthermore, studies from south and southwest India showed no presence of HPV infection [22,23]. All the cases were negative for HPV infection by the CP I/II primer sets. The total HPV positivity by the combined investigation using degenerate primer sets was $12.5 \%$. This rate is lower than that reported for the northeast region of India, where HR-HPV infection was observed to be present in $27.9 \%$ in oral cancer patients [24]. This may be attributed to the loco-regional variance in HPV prevalence.

We also investigated the presence of HPV infection in 75 oropharyngeal cancer cases and observed HPV positivity in $10.7 \%$ of cases by the MY $09 / 11$ primer set and $17.3 \%$ of cases by the GP $5+/ 6+$ primer set. Similar to oral cancer, no positivity was observed using the CP I/II primer set. A study conducted in Delhi reported a 22.8\% HPV infection rate in oropharyngeal cancer cases when analyzed using consensus primers [25]. A total HPV positivity rate of $26.7 \%$ was observed in the cohort. A study conducted in the United States reported that $70 \%$ of cases of oropharyngeal cancers were attributable to HPV infection [26], while a study in Karnataka reported no prevalence of HPV in oropharyngeal cancers [27]. HPV prevalence was observed to be low in both oral and oropharyngeal cancer patients from the western region of India, as the relatively high rate of incidence for both these cancers is attributable to the high consumption of tobacco in this region. Hence, the majority of cancer cases result from tobacco-related oncogenesis rather than HPV infection [28,29].

The study demonstrated a high rate of HPV prevalence (87.0\%) in cervical cancer patients. Moreover, we also observed a notable HPV prevalence (12.5 and 26.7\%) in oral and oropharyngeal cancers, respectively. HPV-positive cancers represent a distinct molecular and clinical entity from HPV-negative cancers [26,27,30,31]. Furthermore, separate guidelines have been added for the staging of HPV-positive oropharyngeal cancers in the eighth edition of the American Joint Committee on Cancer cancer staging guidelines. These guidelines are anticipated to provide a much more accurate and reasonable prediction of survival for newly diagnosed patients [32]. Furthermore, the kappa index between the primer sets was very low, showing no agreement between the primer sets. Thus, it is imperative to screen the population using all 3 primer sets for actual detection. Furthermore, it is especially important to study the HPV burden in HPV-associated cancers with a focus on HR-HPV types. This will help improve the differentiation of $\mathrm{HPV}$-associated cancers and result in a better diagnostic approach for disease management. The screening module with degenerate primer sets (MY 09/11, GP 5+/6+ and CP I/II) used in the present study will aid in screening a higher rate of HR-HPV infections.

In conclusion, the present investigation provides vital data for the study of the burden of HPV-associated cancers. The study found a high rate of HPV infection in cervical cancer and a notable prevalence of HPV infection in oral and oropharyngeal cancers using degenerate primers. Thus, the present study demonstrates the use of a comprehensive screening module for the effective detection of HPV infection in these 3 major malignancies without bias towards any HPV type.

\section{CONFLICT OF INTEREST}

The authors have no conflicts of interest to declare for this study.

\section{FUNDING}

This research was supported by the Gujarat Cancer Society and the Gujarat Cancer and Research Institute for the project (EC/31/ 2018).

\section{ACKNOWLEDGEMENTS}

None. 


\section{AUTHOR CONTRIBUTIONS}

Conceptualization: PSP, PSD, CDP, SDB. Data curation: ART, KAP, KAM. Formal analysis: ART, KAP, PSP. Funding acquisition: PSP, PSD, CDP, SDB. Methodology: ART, KAP. Project administration: PSP, PSD, CDP, SDB. Visualization: PSP, PSD. Writing - original draft: ART, KAP, SDB, KAM, CDP, PSD. Writing review \& editing: PSP.

\section{ORCID}

Ashi Robert Thobias: https://orcid.org/0000-0002-9407-3766; Kinjal Ankit Patel: https://orcid.org/0000-0001-9923-9481; Supreet Dhananjay Bhatt: https://orcid.org/0000-0001-5462-5995; Kruti Ashvinkumar Mehta: https://orcid.org/0000-0001-6921-1234; Chetana Deepal Parekh: https://orcid.org/0000-0003-4811-5889; Pariseema Sharad Dave: https://orcid.org/0000-0003-3300-4414; Prabhudas Shankarbhai Patel: https://orcid.org/0000-0003-10047024

\section{REFERENCES}

1. Bansal A, Singh MP, Rai B. Human papillomavirus-associated cancers: a growing global problem. Int J Appl Basic Med Res 2016; 6:84-89.

2. Nour NM. Cervical cancer: a preventable death. Rev Obstet Gynecol 2009;2:240-244.

3. Petrosky E, Bocchini JA Jr, Hariri S, Chesson H, Curtis CR, Saraiya $\mathrm{M}$, et al. Use of 9-valent human papillomavirus (HPV) vaccine: updated HPV vaccination recommendations of the advisory committee on immunization practices. MMWR Morb Mortal Wkly Rep 2015;64:300-304.

4. Meites E, Szilagyi PG, Chesson HW, Unger ER, Romero JR, Markowitz LE. Human papillomavirus vaccination for adults: updated recommendations of the Advisory Committee on Immunization Practices. Am J Transplant 2019;19:3202-3206.

5. Sharma SJ, Wagner S, Reder HS, Kroll T, Wuerdemann N, Klussmann JP, et al. The 8th edition AJCC/UICC TNM staging for p16-positive oropharyngeal carcinoma: is there space for improvement? Eur Arch Otorhinolaryngol 2018;275:3087-3091.

6. Westra WH. Detection of human papillomavirus (HPV) in clinical samples: evolving methods and strategies for the accurate determination of HPV status of head and neck carcinomas. Oral Oncol 2014;50:771-779.

7. Lombard I, Vincent-Salomon A, Validire P, Zafrani B, de la Rochefordière $\mathrm{A}$, Clough $\mathrm{K}$, et al. Human papillomavirus genotype as a major determinant of the course of cervical cancer. J Clin Oncol 1998;16:2613-2619.

8. Bussu F, Ragin C, Boscolo-Rizzo P, Rizzo D, Gallus R, Delogu G, et al. HPV as a marker for molecular characterization in head and neck oncology: looking for a standardization of clinical use and of detection method(s) in clinical practice. Head Neck 2019;
41:1104-1111.

9. de Martel C, Plummer M, Vignat J, Franceschi S. Worldwide burden of cancer attributable to HPV by site, country and HPV type. Int J Cancer 2017;141:664-670.

10. Thobias AR, Patel KA, Gokani R, Parekh C, Desai A, Patel JB, et al. Prevalence of human papilloma virus infection in cervical cancer patients from Western region of India. Indian J Gynecol Oncol 2019;17:1-6.

11. Senapati R, Nayak B, Kar SK, Dwibedi B. HPV Genotypes distribution in Indian women with and without cervical carcinoma: implication for HPV vaccination program in Odisha, Eastern India. BMC Infect Dis 2017;17:30.

12. Chaturvedi AK, Engels EA, Anderson WF, Gillison ML. Incidence trends for human papillomavirus-related and -unrelated oral squamous cell carcinomas in the United States. J Clin Oncol 2008;26: 612-619.

13. Näsman A, Attner P, Hammarstedt L, Du J, Eriksson M, Giraud G, et al. Incidence of human papillomavirus (HPV) positive tonsillar carcinoma in Stockholm, Sweden: an epidemic of viral-induced carcinoma? Int J Cancer 2009;125:362-366.

14. Tinhofer I, Jöhrens K, Keilholz U, Kaufmann A, Lehmann A, Weichert W, et al. Contribution of human papilloma virus to the incidence of squamous cell carcinoma of the head and neck in a European population with high smoking prevalence. Eur J Cancer 2015;51:514-521.

15. Bray F, Ferlay J, Soerjomataram I, Siegel RL, Torre LA, Jemal A. Global cancer statistics 2018: GLOBOCAN estimates of incidence and mortality worldwide for 36 cancers in 185 countries. CA Cancer J Clin 2018;68:394-424.

16. Basu P, Roychowdhury S, Bafna UD, Chaudhury S, Kothari S, Sekhon R, et al. Human papillomavirus genotype distribution in cervical cancer in India: results from a multi-center study. Asian Pac J Cancer Prev 2009;10:27-34.

17. Munjal K, Adamson CS, Rajendran V, Nandedkar S, Cooper K, Evans MF. Human papillomavirus type distribution in invasive cervical cancers from Madhya Pradesh: implications for vaccination programs in central India. Int J Gynecol Pathol 2014;33:531536.

18. Raza SA, Franceschi S, Pallardy S, Malik FR, Avan BI, Zafar A, et al. Human papillomavirus infection in women with and without cervical cancer in Karachi, Pakistan. Br J Cancer 2010;102:16571660.

19. Vinodhini K, Shanmughapriya S, Sanmugham S, Senthikumar G, Das BC, Natarajaseenivasan K. Prevalence of high-risk HPV and associated risk factors in cases of cervical carcinoma in Tamil Nadu, India. Int J Gynaecol Obstet 2012;119:253-256.

20. Chatterjee S, Chattopadhyay A, Samanta L, Panigrahi P. HPV and cervical cancer epidemiology - current status of HPV vaccination in India. Asian Pac J Cancer Prev 2016;17:3663-3673.

21. Remmerbach TW, Brinckmann UG, Hemprich A, Chekol M, Kühndel K, Liebert UG. PCR detection of human papillomavirus of the mucosa: comparison between MY09/11 and GP5+/6+ primer sets. J Clin Virol 2004;30:302-308. 
22. Dalakoti P, Ramaswamy B, Bhandarkar AM, Nayak DR, Sabeena S, Arunkumar G. Prevalence of HPV in oral squamous cell carcinoma in South West India. Indian J Otolaryngol Head Neck Surg 2019;71(Suppl 1):657-664.

23. Laprise C, Madathil SA, Allison P, Abraham P, Raghavendran A, Shahul HP, et al. No role for human papillomavirus infection in oral cancers in a region in southern India. Int J Cancer 2016;138: 912-917.

24. Kumar R, Rai AK, Das D, Das R, Kumar RS, Sarma A, et al. Alcohol and tobacco increases risk of high risk HPV infection in head and neck cancer patients: study from North-East region of India. PLoS One 2015;10:e0140700.

25. Bahl A, Kumar P, Dar L, Mohanti BK, Sharma A, Thakar A, et al. Prevalence and trends of human papillomavirus in oropharyngeal cancer in a predominantly north Indian population. Head Neck 2014;36:505-510.

26. Cook EE, Gershman ST, Kim JJ, Tamimi RM, Klevens RM, Holmes MD. Trends of two HPV-associated cancers in Massachusetts: cervical and oropharyngeal cancer. Cancer Causes Control 2018;29:435-443.
27. Bandhary SK, Shetty V, Saldanha M, Gatti P, Devegowda D, R PS, et al. Detection of human papilloma virus and risk factors among patients with head and neck squamous cell carcinoma attending a tertiary referral centre in South India. Asian Pac J Cancer Prev 2018;19:1325-1330.

28. Niaz K, Maqbool F, Khan F, Bahadar H, Ismail Hassan F, Abdollahi M. Smokeless tobacco (paan and gutkha) consumption, prevalence, and contribution to oral cancer. Epidemiol Health 2017; 39:e2017009.

29. Bhat VS. Human papilloma virus and oropharyngeal cancer-west and the east. Otolaryngol Online J 2018;8:e101.

30. Otter S, Whitaker S, Chatterjee J, Stewart A. The human papillomavirus as a common pathogen in oropharyngeal, anal and cervical cancers. Clin Oncol 2019;31:81-90.

31. Spence T, Bruce J, Yip KW, Liu FF. HPV associated head and neck cancer. Cancers (Basel) 2016;8:75.

32. Lydiatt WM, Patel SG, O'Sullivan B, Brandwein MS, Ridge JA, Migliacci JC, et al. Head and neck cancers-major changes in the American Joint Committee on cancer eighth edition cancer staging manual. CA Cancer J Clin 2017;67:122-137. 